\title{
Study on the Interaction between BSA and a Water-Soluble Cationic Poly(heteroarylene methine) Bearing Pyridinium Salts
}

\author{
Masaru Sakamaki, Akinori Kato and Yasumasa Fukushima* \\ Department of Biological Applied Chemistry, Graduate School of Engineering, \\ Toyo University, 2100 Kujirai, Kawagoe, Saitama 350-8585, Japan \\ *E-mail:fukusima_m@toyo.jp
}

\begin{abstract}
The interaction of BSA and a water-soluble cationic poly(heteroarylene methine) bearing pyridinium salts (WSCP) has been investigated by spectrophotometric and spectrofluorometric methods. The absorption and fluorescence spectra of BSA suggest that BSA and WSCP form a protein-polymer ground state complex due to electrostatic interaction between the negatively charged surface of BSA and the cationic side chains of WSCP. The interaction causes a decrease in fluorescence intensity of BSA due to static quenching. In addition, an enhancement in fluorescence signal of WSCP with BSA can be attributed to the surfactant property of BSA. The complex formation between BSA and WSCP results from not only electrostatic attractive force but also hydrophobic interaction, and WSCP can become a fluorescence probe for homogeneous BSA detection.
\end{abstract}

Keywords : water-soluble, cationic, poly(heteroarylene methine), pyridinium salt, BSA

\section{Introduction}

Protein is one of the most important bioactive macromolecules, which is related to metabolism, immunity and signal transduction. Accurate and rapid measurement of protein concentration in body is extremely significant in the deamination clinical diagnostics, life sciences and chemical investigation. Therefore there is a high requirement for an effective method to qualify the concentration of specific proteins in environmental and biological samples. Traditional techniques of detecting proteins are generally colorimetric assays such as Bradford method [1], Lowry method [2], and Biuret method [3]. These methods can be easily adapted due to their simple operation, but have limitations such as low sensitivity and narrow linear range. Fluorescence analysis of proteins has attracted considerable attention as a conventional and highly sensitive detecting method $[4,5]$.

Serum albumin, is one of the most abundant soluble carrier proteins in organisms, plays an critical role in the transport and distribution of endogenous and exogenous substances such as amino acids, fatty acids, drugs and steroids in blood plasma [6, 7]. Among the serum albumins, bovine serum albumin (BSA) has been widely studied as a model system because its primary and tertiary structures are determined $[8,9]$. BSA has many biochemical applications due to sequence homology with human serum albumin (HSA). BSA is a globular protein composed of a single polypeptide of 582 amino acids [10]. BSA has two tryptophan residues, Trp-134 and Trp-212. The fluorescence quenching of BSA has been used as a tool to study the interaction between BSA and bioactive small molecules such as surfactants and drugs $[11,12]$. The isoelectric point value of BSA is about 4.7; therefore, the protein displays a negative net charge at neutral $\mathrm{pH}$ [13].

Water-soluble conjugated polymers possess both intriguing optoelectrical properties and good water-solubility, which are versatile materials for various applications such as sensors and electrooptic devices. In particular, conjugated polymers are more advantageous than monomeric fluorescent dyes in highly sensitive fluorescence 
sensor applications due to strong absorption and emission by their delocalized electronic structure [14].

Poly(heteroarylene methine)s, are a kind of conjugated polymers, which are promising materials since they are low band gap polymers which have attractive a great deal of attention due to their intrinsic electronic, optoelectronic, optical and nonlinear properties [15]. Little study has been reported about water-soluble poly(heteroarylene methine)s, although water-soluble conjugated polymers have been extensively investigated for chemical and biological sensing applications.

BSA has been widely used as a typical protein for study on the interaction with biologically important small molecules such as drugs and surfactants. In addition, the interaction of a protein with a water-soluble polymer has also attracted considerable attention in the binding mode and mechanism between a protein and a biomacromolecule in vivo. We demonstrated to interact the cationic water-soluble poly(heteroarylene methine) bearing a quaternary pyridinium group (WSCP) illustrated in Scheme 1 with DNA [16]. However, as far as we are aware, there has been no study on the interaction of poly(heteroarylene methine) with a protein. In this communication, we describe a spectroscopy study of the interaction between negatively charged BSA and WSCP in neutral aqueous solution.

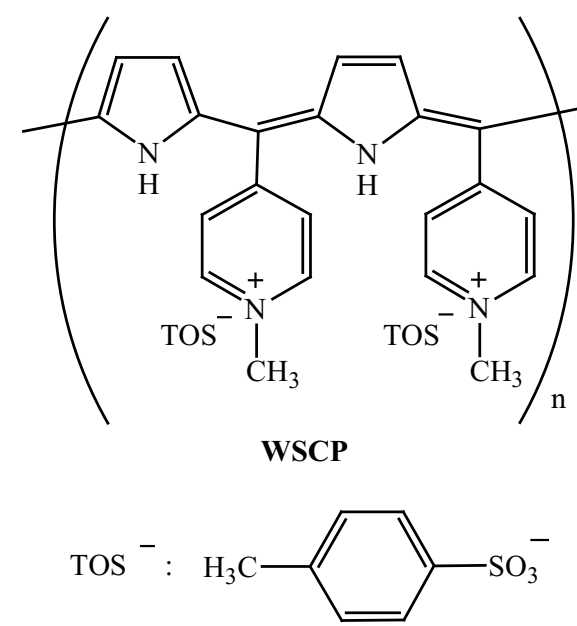

Scheme 1. Structure of water-soluble cationic poly (heteroarylene methine) (WSCP).

\section{Experimental}

\subsection{Materials}

BSA was purchased from Wako Pure Chemical Industries, Ltd. without further purification. Water-soluble cationic poly(heteroarylene methine) (WSCP) was synthesized as previously reported
[16]. All other reagents were of analytical grade unless specified otherwise. The water used was deionized and distilled.

\subsection{Apparatus}

Absorption and fluorescence spectra were recorded on a JASCO UV-530 spectrophotometer and a JASCO FP-750 spectrofluorometer, respectively. The $\mathrm{pH}$ was measured using a HANNA instruments $\mathrm{pH} 211 \mathrm{pH}$ meter.

\subsection{Procedure}

BSA stock solution $(100 \mu \mathrm{M})$ was prepared by dissolving a certain amount of BSA in water and stored in refrigerator. The concentration of WSCP stock water solution was $1000 \mu \mathrm{M}$ as monomer unit concentration. Tris- $\mathrm{HCl}$ buffer solution was used to control the $\mathrm{pH}$ of the measured solution. In a $5 \mathrm{~mL}$ volumetric flask, $50 \mu \mathrm{L}$ of $1 \mathrm{M}$ Tris- $\mathrm{HCl}$ buffer solution (pH 6.8), and appropriate volumes of BSA and WSCP stock solutions, respectively, were added. The mixture was diluted with water to the scale and mixed homogeneously. After $15 \mathrm{~min}$ at room temperature, the absorption and fluorescence spectra were measured.

\section{Results and discussion}

Under the experimental conditions, BSA naturally has fluorescence and their maximum excitation and emission wavelengths are at 280 and $340 \mathrm{~nm}$, respectively. This fluorescence is mainly attributed to the presence of Trp and Tyr residues $[17,18]$. The interaction between BSA and WSCP was investigated by evaluating fluorescence intensity on the BSA before and after the addition of WSCP. The effect of WSCP on BSA fluorescence intensity is shown in Fig. 1. The fluorescence intensity of BSA decreased regularly along with the increase in the WSCP concentration but the maximum fluorescence wavelength was not shifted. This indicates that WSCP could interact with BSA and quench its intrinsic fluorescence, but there was no change in the local environment of BSA. WSCP contains a pyridinium moiety which is an efficient fluorescence quencher that could reduce the intrinsic protein fluorescence [19]. It can be expected that this quenching efficiency would increase if the local WSCP concentration is increased by its interaction with BSA.

Fluorescence quenching efficiencies can be analyzed by the use of the Stern-Volmer equation (1) [20]:

$$
\mathrm{I}_{0} / \mathrm{I}=1+K_{\mathrm{SV}}[\mathrm{Q}]=1+K_{\mathrm{q}} \tau_{0}[\mathrm{Q}]
$$


where the $I_{0}$ and $I$ are the fluorescence intensities of BSA solutions at $340 \mathrm{~nm}$ in the absence and presence of WSCP with various concentrations, respectively. $K_{\mathrm{SV}}$ is the Stern-Volmer dynamic quenching constant and [Q] is the concentration of WSCP. $K_{\mathrm{q}}$ is the dynamic quenching rate constant, $\ddot{A}$ is the average lifetime of the fluorophore in the absence of quencher. The quenching constant can provide a direct measure of the quenching efficiency and be obtained from linear portion of the slope in the Stern-Volmer plots.

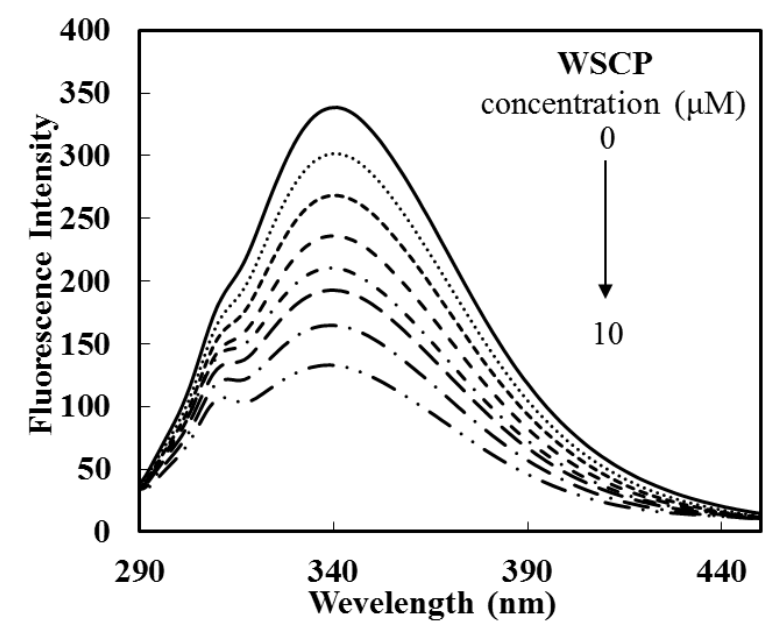

Fig. 1. Fluorescent titration spectra of BSA $(0.2 \mu \mathrm{M})$ in $10 \mathrm{mM}$ Tris- $\mathrm{HCl}$ buffer at $\mathrm{pH} 6.8$ in the presence of WSCP at different concentrations, [WSCP] $=0,1,2,3$, $4,5,7$, and $10 \mu \mathrm{M}$ upon excitation at $280 \mathrm{~nm}$.

At WSCP concentration ranging from 0 to 10 $\mu \mathrm{M}$, a linear plot with $K \mathrm{sv}=1.52 \times 10^{5} \mathrm{M}^{-1}\left(\mathrm{R}^{2}=\right.$ 0.996) was obtained. It is reported that $\tau_{0}$, the average fluorescence lifetime of biopolymer is about $10^{-8} \mathrm{~s}$, the dynamic quenching rate constant $K_{\mathrm{q}}$ is calculated to be $1.52 \times 10^{13} \mathrm{M}^{-1} \cdot \mathrm{s}^{-1}$ [21]. Dynamic and static quenching can be distinguished by the quenching constant $K_{\mathrm{q}}$. Dynamic quenching refers to a process that a fluorophore and a quencher collide during the lifetime of the excited state, whereas static quenching refers to ground state complex formation between a fluorophore and a quencher. For dynamic quenching, $K_{\mathrm{q}}$ cannot exceed the limiting diffusion rate constant of the biomolecule (ca. $2 \times 10^{10} \mathrm{M}^{-1} \cdot \mathrm{s}^{-1}$ ). The obtained value of $K_{\mathrm{q}}$ was much larger than $2 \times 10^{10} \mathrm{M}^{-1} \cdot \mathrm{s}^{-1}$. This result suggests that static quenching dominates the quenching mechanism, while dynamic quenching could be negligible in the concentration range studied. Because BSA and WSCP are oppositely charged, they would form ground state complex by electrostatic interaction.
For confirming the complex formation between BSA and WSCP, we have measured the absorption spectra of BSA with and without WSCP in aqueous solution. Figure 2 displays the absorption spectra of BSA, WSCP, the sum of their spectra, and the mixture of BSA and WSCP. The absorption spectrum of BSA with WSCP is much larger than the sum of two spectra of BSA and WSCP, resulting in their complex formation [22].

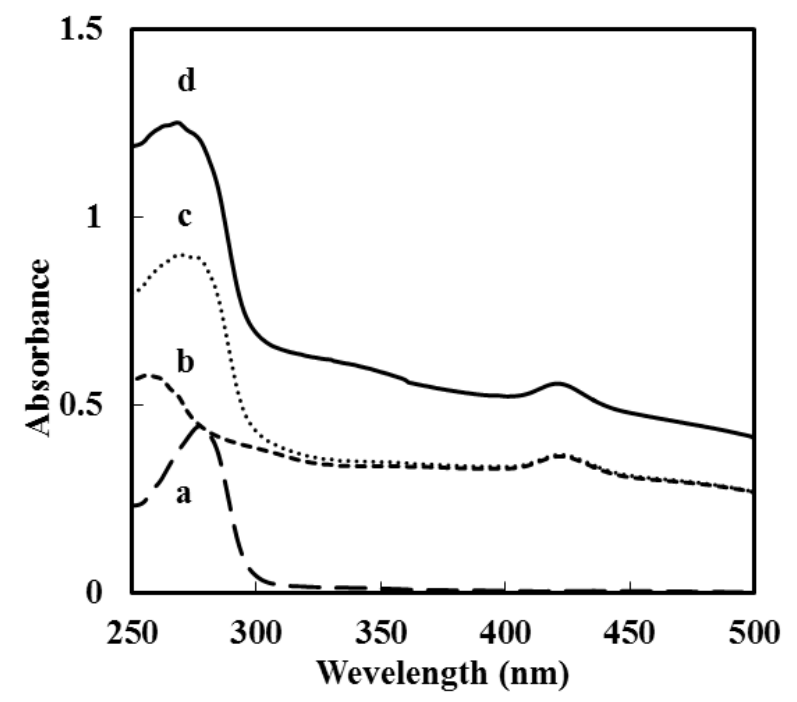

Fig. 2. Absorption spectra of (a) BSA $(10 \mu \mathrm{M})$, (b) WSCP $(100 \mu \mathrm{M})$, (c) the sum of BSA $(10 \mu \mathrm{M})$ and WSCP $(100 \mu \mathrm{M})$, and (d) the mixture solution of BSA $(10 \mu \mathrm{M})$ and WSCP $(100 \mu \mathrm{M})$, in $10 \mathrm{mM}$ Tris- $\mathrm{HCl}$ buffer at $\mathrm{pH} 6.8$.

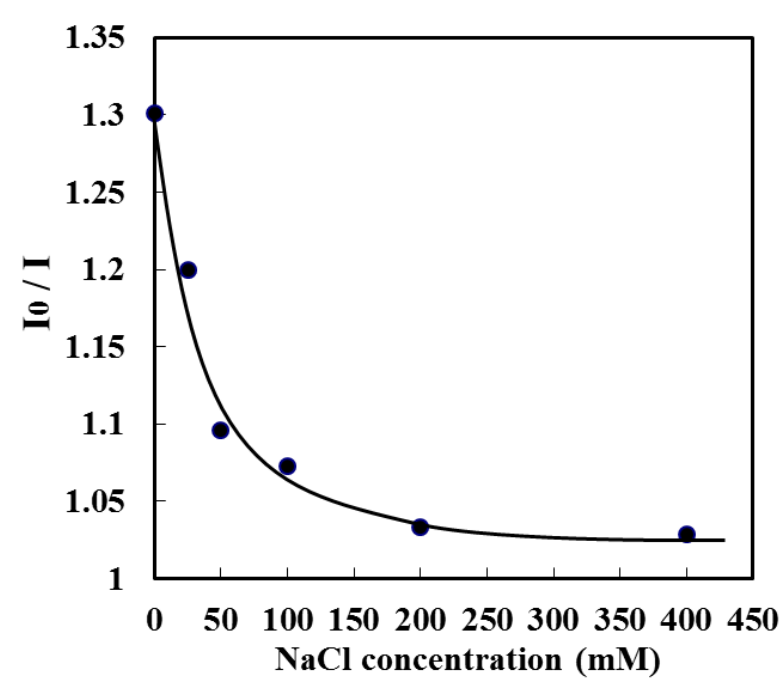

Fig. 3. The relative fluorescence intensity $\left(\mathrm{I}_{0} / \mathrm{I}\right)$ at 340 $\mathrm{nm}$ for BSA $(0.2 \mu \mathrm{M})$ as a function of $\mathrm{NaCl}$ concentration in $10 \mathrm{mM}$ Tris- $\mathrm{HCl}$ buffer at $\mathrm{pH} 6.8$ in the presence of WSCP $(10 \mu \mathrm{M})$ upon excitation at $280 \mathrm{~nm}$.

WSCP carries positive charges on pyridinium groups, while BSA displays a negative net charge 
in the $\mathrm{pH} 6.8$ Tris-HCl buffer because its isoelectric point value is about 4.7. WSCP can interact with BSA through electrostatic forces. Figure 3 shows that the study of the interaction between WSCP and BSA was performed by measuring the changes of the relative fluorescence intensity in various ionic concentrations. The relative fluorescence intensity $\left(\mathrm{I}_{0} / \mathrm{I}\right)$ decreases with $\mathrm{NaCl}$ concentration. This result indicates that the interaction between WSCP and BSA becomes weaker due to electrostatic shielding with increasing the ionic strength. Because the complex formation is significantly affected by the ionic strength, the electrostatic interaction between WSCP and BSA plays an important role in the complex formation.
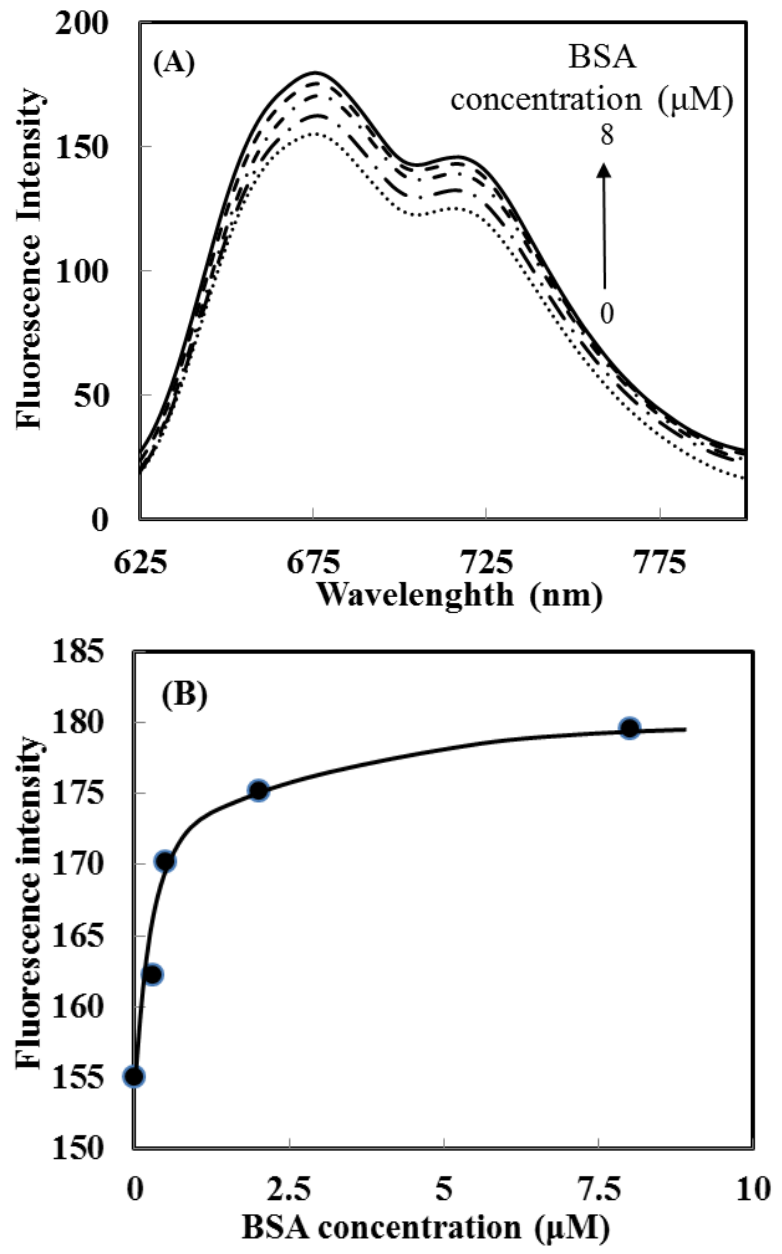

Fig. 4. (A)Fluorescent titration spectra of WSCP (100 $\mu \mathrm{M})$ in $10 \mathrm{mM}$ Tris- $\mathrm{HCl}$ buffer at $\mathrm{pH} 6.8$ in the presence of BSA at different concentrations, $[\mathrm{BSA}]=0$, $0.3,0.5,2$, and $8 \mu \mathrm{M}$ upon excitation at $424 \mathrm{~nm}$.

(B) Fluorescence intensity at $675 \mathrm{~nm}$ of WSCP as a function of BSA concentration.

The fluorescence response of WSCP toward BSA was investigated in $10 \mathrm{mM}$ Tris- $\mathrm{HCl}$ buffer at
pH 6.8. Figure 4(A) shows the fluorescence spectra of WSCP at $100 \mu \mathrm{M}$ in the presence of BSA with the BSA concentration ranging from 0 to $8 \mu \mathrm{M}$ upon excitation at $424 \mathrm{~nm}$. WSCP emits fluorescence ranging from 625 to $800 \mathrm{~nm}$ with two peak maxima around 680 and $720 \mathrm{~nm}$. WSCP exhibits fluorescence enhancement upon BSA addition but the maximum fluorescence wavelength was not shifted. It has been widely reported that BSA, a fatty acid transporter with surfactant effect, often leads to an increase in fluorescence [23]. The increased fluorescence of WSCP upon addition of BSA suggests that the polymer backbone forms efficiently complex between BSA and WSCP by the hydrophobic patches on the surface of BSA. The fluorescence spectral variations of WSCP upon BSA addition are thus due to the increased hydrophobicity of WSCP environment that reduces the contact between the water molecules and the electron-delocalized conjugated backbone.

As shown in Figure 4(B), the fluorescence intensity increases drastically up to $0.5 \mu \mathrm{M}$ of BSA concentration and enhances gradually above 0.5 $\mu \mathrm{M}$. In addition to the electrostatic attractive interaction, hydrophobic interaction could be one of the important forces in the molecular interaction between WSCP and BSA.

\section{Conclusion}

In conclusion, we have investigated the interaction between BSA and WSCP from changes observed in their absorption and fluorescence behaviors. The fluorescence of BSA can be quenched by WSCP at its micromolar concentrations where their ground state complex forms by electrostatic attractive force between the positively charged pyridinium moieties of WSCP and negatively charged BSA in neutral aqueous solution. On the other hands, the fluorescence of WSCP increases a little by the addition of BSA at its sub-micromolar concentrations where the surfactant property of BSA can lead to their complex formation. Therefore, the complex between BSA and WSCP forms by electrostatic interaction as well as hydrophobic interaction, and a water-soluble cationic conjugated polymer containing a pyridinium salt is expected to have potential applications in various biomolecular detections.

\section{References}

1. M.M. Bradford, Anal. Biochem., 72 (1976) 248.

2. O.H. Lowry, N.J. Rosebrough, A.L. Farr, R.J. Randall, J. Biol. Chem., 193 (1951) 265. 
3. F. Joseph, Biochem. J., 29 (1935) 799.

4. C.A. Royer, Chem. Rev., 106 (2006) 1769.

5. A.P. deSilva, H.Q.N. Gunaratne, T. Gunnlaugsson, A.J.M. Huxley, C.P. McCoy, J.T.

Rademacher, T.E. Rice, Chem. Rev., 97 (1997) 1515.

6. W.E. Muller, U. Wollert, Pharmacology, 19 (1979) 59.

7. R.E. Olson, D.D. Christ, Annu. Rep. Med. Chem., 31 (1996) 327.

8. A. Papadopoulou, R.J. Green, R.A. Frazier, J. Agric. Food Chem., 53 (2005) 158.

9. J. Min, J.W. Lee, Y.H. Ahn, Y.T. Chang, J. Comb. Chem., 9 (2007) 1079.

10. F.P. Nicoletti, B.D. Howes, M. Fittipaldi, G. Fanali, M. Fasano, P. Ascenzi, G. Smulevich, J. Am. Chem. Soc., 130 (2008) 11677.

11. J.S. Johansson, R.G. Eckenhoff, P.L. Dutton, Anesthesiology, 83 (1995) 316.

12. D. Silva, C.M. Cortez, S.R.W. Louro, Spectrochim. Acta. A, 60 (2004) 1215.

13. Q. Shi, Y. Zhou, Y. Sun, Biotechnol. Prog., 21 (2005) 516.

14. S.W. Thomas, G.D. Joly, T.M. Swager, Chem.
Rev., 107 (2007) 1339.

15. W. Chen, C. Liu, C. Yen, F. Tsai, C.J. Tonzola, N, Olson, S.A. Jenekhe, Macromolecules, 37 (2004) 5959.

16. A. Kato, Y. Fukushima, J. Photopolym. Sci. Technol., 26 (2013) 171.

17. B.F. Pan, F. Gao, L.M. Ao, J. Magn. Magn. Mater., 293 (2005) 252.

18. K.A.Z. Osama, I.K.A.S.H. Othman, J. Am. Chem. Soc., 130 (2008) 1079.

19. L. Miola, R. Abakerll, M.F. Ginani, P.B. Filho, V.G. Toscano, F.H. Quina, J. Phys. Chem., 87 (1983) 4417.

20. J.R. Lakowicz, "Principles of Fluorescence Spectroscopy", Plenum Press, New York (1983) p257.

21. J.R. Lakowicz, G. Weber, Biochemistry, 12 (1973) 4161.

22. K.S. Witold, H.M. Henry, C. Dennis, Biochemistry, 32 (1993) 389.

23. J.J. Lavigne, D.L. Broughton, J.N. Wilson, B. Erdogan, B. Bunz, Macromolecules, 35 (2003) 7409. 